\title{
Effects of nanoscale spatial inhomogeneity in strongly correlated systems
}

\author{
M. F. Silva, ${ }^{1}$ N. A. Lima, ${ }^{2}$ A. L. Malvezzi, ${ }^{3}$ and K. Capelle ${ }^{4, *}$ \\ ${ }^{1}$ Instituto de Física, Universidade de São Paulo, Caixa Postal 66318, 05315-970 São Paulo, SP, Brazil \\ ${ }^{2}$ Colegiado de Engenharia de Produção, Fundação Universidade Federal do Vale do São Francisco, Caixa Postal 252, \\ 56306-410 Petrolina, PE, Brazil \\ ${ }^{3}$ Departamento de Física, Faculdade de Ciências, Universidade Estadual Paulista, Caixa Postal 473, 17015-970 Bauru, SP, Brazil \\ ${ }^{4}$ Departamento de Física e Informática, Instituto de Física de São Carlos, Universidade de São Paulo, Caixa Postal 369, \\ 13560-970 São Carlos, SP, Brazil
}

(Received 10 January 2005; published 29 March 2005)

\begin{abstract}
We calculate ground-state energies and density distributions of Hubbard superlattices characterized by periodic modulations of the on-site interaction and the on-site potential. Both density-matrix renormalization group and density-functional methods are employed and compared. We find that small variations in the on-site potential $v_{i}$ can simulate, cancel, or even overcompensate effects due to much larger variations in the on-site interaction $U_{i}$. Our findings highlight the importance of nanoscale spatial inhomogeneity in strongly correlated systems, and call for a reexamination of model calculations assuming spatial homogeneity.
\end{abstract}

DOI: 10.1103/PhysRevB.71.125130 PACS number(s): 71.10.Fd, 71.10.Pm, 71.15.Mb, 71.27.+a

A large part of the complexity of strongly correlated systems arises from the multiple phases that coexist or compete in their phase diagrams. Metallic and insulating phases are separated by metal-insulator transitions, and subject to the formation of various types of long-range order, such as antiferromagnetism, superconductivity, and charge- or spindensity waves. The relative stability of such phases is determined by differences in appropriate thermodynamic potentials, or, at zero temperature, in their ground-state energies. Identification of the appropriate order parameters and calculation of the ground-state energies of the various phases is a complicated problem, and the nature of the phase diagram of many strongly correlated systems is still subject to considerable controversy. It is widely believed, however, that a minimal model containing the essence of strong correlations, and displaying many of the above-mentioned phases, is the homogeneous Hubbard model, which in one dimension and standard notation reads

$$
\hat{H}_{h o m}=-t \sum_{i, \sigma}\left(c_{i \sigma}^{\dagger} c_{i+1, \sigma}+\text { H.c. }\right)+U \sum_{i} c_{i \uparrow}^{\dagger} c_{i \uparrow} c_{i \downarrow}^{\dagger} c_{i \downarrow} .
$$

Much theoretical effort is thus going into the analysis of the homogeneous Hubbard model and the clarification of the nature of its ground state.

In a parallel development, nanoscale spatial inhomogeneity has been observed experimentally to be a ubiquitious feature of strongly correlated systems, ${ }^{1-8}$ but although its importance is widely recognized, the consequences of such inhomogeneity are still insufficiently understood. The present paper investigates the effects of, and the competition between, two different manifestations of nanoscale inhomogeneity in strongly correlated systems, namely local variations in the on-site potential and in the on-site interaction. We base our analysis on the inhomogeneous Hubbard model

$$
\begin{aligned}
\hat{H}_{\text {inhom }}= & -t \sum_{i, \sigma}\left(c_{i \sigma}^{\dagger} c_{i+1, \sigma}+\text { H.c. }\right)+\sum_{i} U_{i} c_{i \uparrow}^{\dagger} c_{i \uparrow} c_{i \downarrow}^{\dagger} c_{i \downarrow} \\
& +\sum_{i \sigma} v_{i} c_{i \sigma}^{\dagger} c_{i \sigma},
\end{aligned}
$$

which differs from the homogeneous model (1) by allowing for spatial variations in the on-site interaction $U_{i}$ and the presence of the on-site potential $v_{i}$. Variations in $U_{i}$ and $v_{i}$ may arise, e.g., due to inequivalent sites in the natural unit cell, modulation of system parameters in artificial heterostructures, or self-consistent modulations in local system properties due to formation of charge-ordered states. In this paper, we are specifically concerned with one-dimensional superlattice structures in which both $U_{i}$ and $v_{i}$ vary periodically on a length scale comparable to, or somewhat larger than, the lattice constant. Such superlattices have recently attracted much attention due to their complex ground-state and transport properties. ${ }^{9-18}$ Our results, reported below, have a direct bearing on the investigation of such superlattices. However, for our present purposes the most important aspect of superlattice structures is that they constitute a representative system in which the consequences of nanoscale spatial variations of system parameters in the presence of strong Coulomb correlations can be explored systematically. Accordingly, we expect our main conclusions to hold also in many other spatially inhomogeneous correlated systems.

Figure 1 shows the density profile of a typical superlattice structure in which the on-site interaction $U_{i}$ is modulated in a repeated pattern of repulsive $\left(U_{i}=3\right)$ and noninteracting $\left(U_{i}=0\right)$ "layers" with $L_{U}$ and $L_{0}$ sites, respectively, and the on-site potential $v_{i}$ is taken to be constant at all sites. The two curves shown were obtained with different many-body techniques. The full curve was obtained using the densitymatrix renormalization group (DMRG),${ }^{19,20}$ while the dotted curve was obtained from density-functional theory (DFT) within the Bethe-Ansatz local-density approximation (BA-LDA). ${ }^{21-23}$ In view of the complexity of the problem 


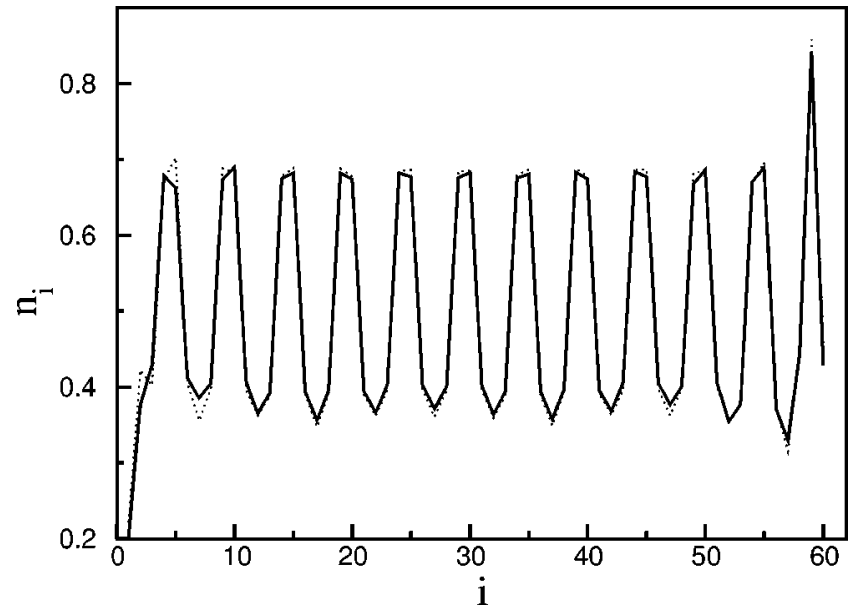

FIG. 1. Density profile of a one-dimensional superlattice with $L=60$ sites, $N=30$ fermions, open boundary conditions, and a superlattice structure consisting of a periodic sequence of $L_{U}=3$ interacting $\left(U_{i}=3\right)$ and $L_{0}=2$ noninteracting $\left(U_{i}=0\right)$ sites. Full curve: DMRG calculation. Dotted curve: DFT/BA-LDA calculation.

and the surprising nature of some of our conclusions, we found it advisable to bring two independently developed and implemented many-body methods to bear on the problem.

DMRG is a well-established numerical technique, whose precision can be improved systematically, at the expense of increased computational effort. ${ }^{19,20}$ In our DMRG calculations, truncation errors were kept of the order of $10^{-6}$ or smaller, and increasing the precision beyond this did not affect any of our conclusions. BA-LDA is a more recent development $^{21-23}$ (although the original LDA concept is, of course, widely used in ab initio calculations). In LDA calculations, the final precision is ultimately limited by the locality assumption inherent in the LDA, and improvements must come from the development of better functionals. This intrinsic limitation of LDA is offset by its applicability to very large and inhomogeneous systems, at much reduced computational effort: Calculations for the type of superlattice structures investigated here typically take only seconds to minutes with BA-LDA, regardless of the type of boundary condition used. ${ }^{24}$ Final BA-LDA results for densities and energies typically agree with DMRG ones to within $\$ 3 \%$, the agreement being slightly better for energies than for densities. ${ }^{25}$ Here we consider both methods as complementary. All essential conclusions reported below were obtained on the basis of independently implemented and performed BA-LDA and DMRG calculations. As an illustration, Table I compares ground-state energies obtained with both methods for one much larger and one much more rapidly modulated superlattice than the one shown in Fig. 1.

Inspection of Fig. 2 shows that an attractive potential on the repulsively interacting sites can completely reverse the effect of the Coulomb repulsion $U_{i}$ and draw a substantial number of electrons to the interacting sites (circles in Fig. 2). While this might have been anticipated qualitatively as a result of the competition between an attraction and a repulsion, it comes as a surprise that the effect of the (often neglected) variations in the on-site potential is much stronger than the one of variations in the on-site interaction: already a
TABLE I. Ground-state energy of two open superlattices with modulated on-site interaction $U_{i}$ and spatially constant on-site potential $v_{i}$, obtained with DMRG and with DFT/BA-LDA. Upper part: large lattice with $L=300$ sites. $L_{U}=10$ interacting sites $\left(U_{i}\right.$ $=3$ ) alternate with $L_{0}=10$ noninteracting sites $\left(U_{i}=0\right)$. Lower part: strongly modulated lattice with $L=100$ sites. $L_{U}=1$ interacting site $\left(U_{i}=6\right)$ alternates with $L_{0}=1$ noninteracting site. $N$ is the number of fermions, and the column labeled $\Delta \%$ contains the absolute percent deviation of the DMRG from the DFT/BA-LDA values. The agreement between DMRG and BA-LDA is slightly better for the more slowly modulated lattice. ${ }^{25}$

\begin{tabular}{llll}
\hline \hline$N$ & $E_{0}^{\mathrm{DMRG}} / t$ & $E_{0}^{\mathrm{BA}-\mathrm{LDA}} / t$ & $\Delta \%$ \\
\hline 50 & -97.994 & -98.342 & 0.35 \\
100 & -185.66 & -187.32 & 0.89 \\
150 & -255.62 & -258.65 & 1.17 \\
200 & -302.10 & -305.68 & 1.17 \\
250 & -321.09 & -324.02 & 0.90 \\
300 & -310.35 & -311.87 & 0.49 \\
40 & -69.822 & -71.110 & 1.81 \\
50 & -82.078 & -83.730 & 1.97 \\
75 & -100.14 & -103.11 & 2.88 \\
80 & -101.81 & -104.94 & 2.98 \\
120 & -79.947 & -79.323 & 0.79 \\
\hline \hline
\end{tabular}

very weak attractive potential suffices to smooth out the density distribution, resulting in an essentially homogeneous charge profile (triangles in Fig. 2). Although we have taken superlattices as our example, the effect is clearly not dependent on periodicity of the modulations in $U_{i}$ and $v_{i}$, and is expected to show up rather generally.

Figure 1 and Table I represent superlattices in which only the on-site interaction is spatially modulated, which is the case most studied in the literature. ${ }^{9-17}$ Many important features of superlattice structures are already apparent in this type of model. However, in a real system it is impossible to modulate the on-site interaction without simultaneously modulating the on-site potential as well, i.e., without creating inequivalent sites. Such a double modulation is found, e.g., in artificially grown layered structures, in impurity systems, and in periodic arrays of Fermi-liquid leads (corresponding to approximately noninteracting sites) and quantum wires/ dots (corresponding to interacting sites). Figure 2 illustrates the consequences a modulation of the on-site potential has on the density profile of a system in which both interaction and potential vary.

In Fig. 3, we compare the Friedel oscillations arising from the system boundaries in a homogeneous system with the ones arising in a superlattice of the same size and with the same number of fermions, but subject to periodic modulations of $U_{i}$ and $v_{i}$, chosen such that both density profiles become similar. We have deliberately not chosen modulation parameters that optimize the agreement between both curves, because had we done so they would be visually indistinguishable on this scale.

Figure 2 shows that a small value of $v_{i}$ can have stronger effects than a larger value of $U_{i}$, while Fig. 3 shows that 


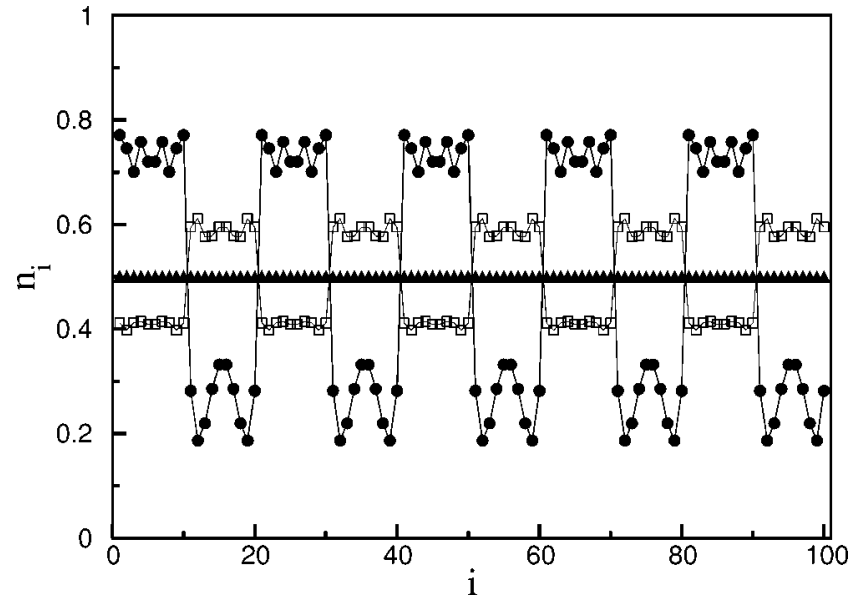

FIG. 2. Density profiles, obtained with BA-LDA, of an $L=100$ site system of $N=50$ fermions with periodically modulated on-site interaction of amplitude $U=3\left(L_{U}=L_{0}=10\right)$ and periodic boundary conditions. ${ }^{26}$ Squares: no modulation in on-site potential $\left(v_{i}=0\right)$. Circles: on-site potential modulated such that $v_{i}=-2$ on the interacting sites and $v_{i}=0$ on the noninteracting sites. The density profile is inverted, indicating overcompensation of $U_{i}$ by $v_{i}$. Triangles: onsite potential modulated such that $v_{i}=-0.555$ on the interacting sites and $v_{i}=0$ on the noninteracting sites. The superlattice structure is erased from the density profile. The lines are guides for the eye.

essentially the same modulation pattern in the density profile can be obtained from either $v$ or $U$. These are unexpected findings. Normally it is assumed that in systems modeled by the Hubbard model, the particle-particle interaction $U$ is much more important than the on-site potential, which is mostly taken to be spatially constant, or, if it varies, to produce only minor modifications in systems whose physics is governed by $U$.

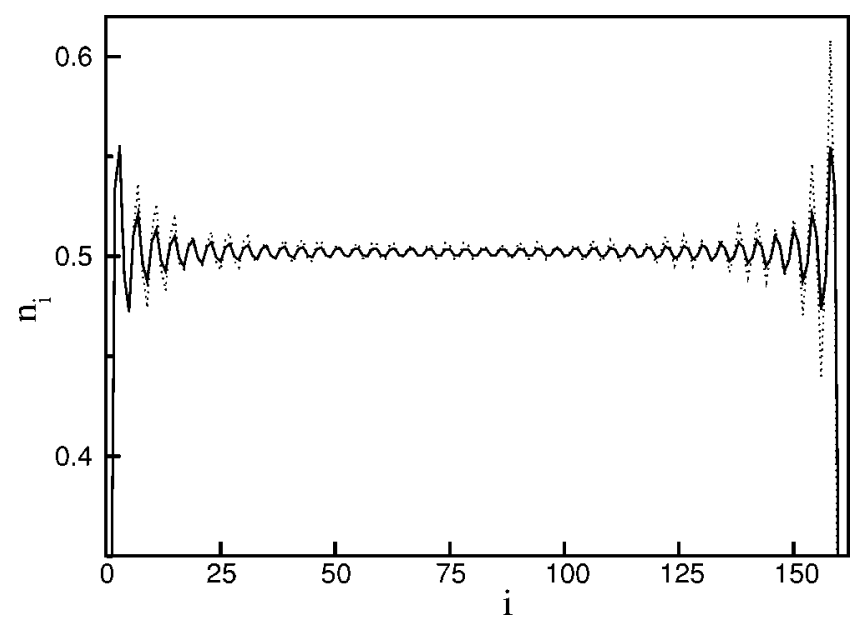

FIG. 3. Full curve: density profile, obtained with BA-LDA, of an $L=160$ site homogeneous system with open boundary conditions, $U_{i}=2, N=80, v_{i}=0$. Friedel oscillations arising from the system boundaries are clearly visible. ${ }^{26}$ Dotted curve: density profile of the same system but subject to modulations periodically alternating $L_{U}=6$ sites with $U_{i}=2$ and $v_{i}=-0.2$, with $L_{0}=10$ sites with $U_{i}=v_{i}$ $=0$. The superlattice structure due to the presence of both modulations is completely erased from the density profile, while the Friedel oscillations arising from the boundary remain prominent.

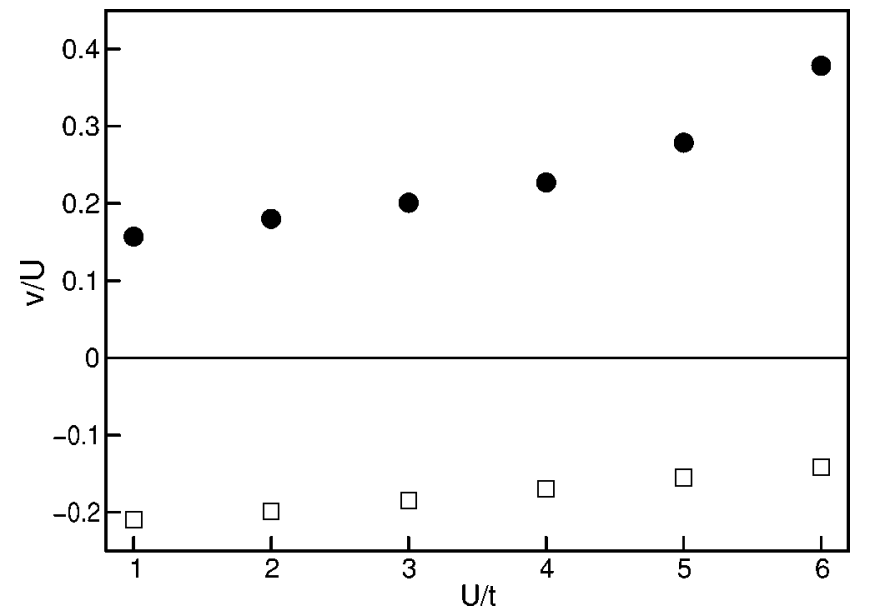

FIG. 4. Open squares: Amplitude of the modulation of the attractive on-site potential that cancels as much as possible the effect of the modulation of the repulsive on-site interaction, leading to a homogeneous density profile [criterion (i)]. Full circles: Amplitude of the modulation of the attractive on-site potential that reproduces in the doubly modulated system the energy of the homogeneous system [criterion (ii)]. System parameters: $L=160$ sites, $N=80$ fermions, $L_{U}=6$ interacting sites, alternating with $L_{0}=10$ noninteracting sites. ${ }^{27}$

To investigate in more detail this competition between on-site interaction and on-site potential, we need to establish criteria for comparing the consequences of $v_{i}$ and of $U_{i}$. Motivated by the experimental observation of nanoscale density variations and by the theoretical importance of ground-state energies for analyses of phase diagrams, we adopt two distinct criteria. Criterion (i) consists in searching for that modulation of the on-site potential $v_{i}$ in a doubly modulated lattice that cancels the effect of the modulation of $U_{i}$ on the density distribution, i.e., smoothes out the oscillations, making the net density homogeneous. A particular example of this cancellation is given by the triangles in Fig. 2. Criterion (ii) consists in searching for that modulation of the on-site potential $v_{i}$ in the doubly modulated lattice that yields the same ground-state energy $E_{0}$ as in a homogeneous lattice with $v_{i}=0$ and $U_{i}=U$ at all sites. ${ }^{27}$ Our results, displayed in Fig. $4,{ }^{24}$ show that, regardless of whether one adopts the density or the energy criterion, the modulation of the on-site potential required to cancel the effect of the modulation of the on-site interaction is up to an order of magnitude smaller than $U$. For open boundary conditions, we have obtained the same conclusion also from DMRG calculations. Changes in the modulation pattern do not change the order of magnitude of the ratio of $|v|$ to $U$ appreciably.

A semiquantitative explanation for this relation of $|v|$ to $U$ can be given within DFT by considering the effective potential entering the Kohn-Sham equations for the Hubbard model, $v_{e f f, i}=v_{e x t, i}+v_{H, i}+v_{c, i}$. For unpolarized systems $\left(n_{\uparrow, i}\right.$ $\left.=n_{\downarrow, i}=n_{i} / 2\right)$, the Hartree potential $v_{H, i}$ can be written $v_{H, i}$ $=U_{i} n_{i} / 2$. Within BA-LDA DFT, the density and total energy are thus calculated from an effective Hamiltonian containing the modulated interaction and external potential only via the combination $v_{e x t, i}+U_{i} n_{i} / 2+v_{c, i}\left(n_{i}, U_{i}\right)$. Since the correlation potential $v_{c, i}$ is typically about an order of magnitude smaller 
than $v_{H, i}$, the modulated interaction $U_{i}$ enters the effective Hamiltonian approximately on the same footing as the modulated potential $v_{\text {ext, } i}$, but renormalized by the factor $n_{i} / 2$. In the above calculations, the average density $N / L$ $=0.5$, and the local density $n_{i}$ is not very different. The upshot is that self-consistent screening of the particle-particle interaction effectively reduces the modulation in the interaction by a factor $\sim 4$, compared to modulations in the potential, in good agreement with the numerical results in Fig. $4 .^{28}$

Of course, in a real system one cannot adjust $v_{i}$ at will, and the precise fine-tuning required to obtain smooth density profiles, or energies identical to the ones found in homogeneous systems, is not expected to occur frequently in nature. The main implication of these criteria is rather that they establish a scale for comparison of $v$ and $U$, indicating that even weak spatial variations of $v$ can be more important than much stronger ones in $U$. This observation flags a warning signal to the use of homogeneous Hubbard models (or ones in which only $U$ is modulated) in the analysis of situations characterized by nanoscale spatial inhomogeneity, such as the pseudogap phase of cuprates ${ }^{1-8}$ or superlattices and similar heterostructures. ${ }^{9-18}$

We conclude that even in the presence of strong correla- tions, spatial variations of the on-site potential $v_{i}$ are not a minor complication in a system dominated by the on-site interaction $U_{i}$, but a major effect, which crucially contributes to observables, and can mask or overcompensate the effect of the interaction on the density profile, ground-state energy, and other quantities. For the density profile, this means that attempts to model the microscopically inhomogeneous charge distribution, seen experimentally, ${ }^{1-8}$ by Hubbard models that are homogeneous or that modulate only the interaction $U_{i}$, cannot lead to conclusive results. The influence of modulations in $v_{i}$ on the ground-state energy, on the other hand, implies that an analysis of the relative energetic stability of the various phases appearing in strongly correlated systems is incomplete, and potentially misleading, if the effects of spatial inhomogeneity in these phases are not taken into account. All this calls into question the common practice to employ the homogeneous Hubbard model to model spatially inhomogeneous many-body systems, and demands a reconsideration of the role of nanoscale spatial inhomogeneity in strongly correlated systems. ${ }^{1-18}$

This work was supported by FAPESP and CNPq.
*Electronic address: capelle@if.sc.usp.br

${ }^{1}$ Intrinsic Multiscale Structure and Dynamics in Complex Electronic Oxides, edited by A. R. Bishop, S. R. Shenoy, and S. Sridhar (Word Scientific, New Jersey, 2003).

${ }^{2}$ K. McElroy, D.-H. Lee, J. E. Hoffman, K. M. Lang, E. W. Hudson, H. Eisaki, S. Uchida, J. Lee, and J. C. Davis, e-print condmat/0404005.

${ }^{3}$ D. J. Derro, E. W. Hudson, K. M. Lang, S. H. Pan, J. C. Davis, J. T. Markert, and A. L. de Lozanne, Phys. Rev. Lett. 88, 097002 (2002).

${ }^{4}$ A. Yazdani, C. M. Howald, C. P. Lutz, A. Kapitulnik, and D. M. Eigler, Phys. Rev. Lett. 83, 176 (1999).

${ }^{5}$ E. W. Hudson, S. H. Pan, A. K. Gupta, K.-W. Ng, and J. C. Davis, Science 285, 88 (1999).

${ }^{6}$ K. M. Lang, V. Madhavan, J. E. Hoffman, E. W. Hudson, H. Eisaki, S. Uchida, and J. C. Davis, Nature (London) 415, 412 (2002).

${ }^{7}$ S. H. Pan, J. P. O’Neal, R. L. Badzey, C. Chamon, H. Ding, J. R. Engelbrecht, Z. Wang, H. E. Eisaki, S. Uchida, A. K. Gupta, K.-W. Ng, E. W. Hudson, K. M. Lang, and J. C. Davies, Nature (London) 413, 282 (2001).

${ }^{8}$ T. Cren, D. Roditchev, W. Sacks, and J. Klein, Europhys. Lett. 54, 84 (2001).

${ }^{9}$ A. L. Malvezzi, T. Paiva, and R. R. dos Santos, Phys. Rev. B 66, 064430 (2002); [Virtual Journal of Nanoscale Science \& Technology Vol. 6 (2002) (www.vinano.org)].

${ }^{10}$ J. Silva-Valencia, E. Miranda, and R. R. dos Santos, Phys. Rev. B 65, 115115 (2002).

${ }^{11}$ J. Silva-Valencia, E. Miranda, and R. R. dos Santos, J. Phys.: Condens. Matter 13, L619 (2001).

${ }^{12}$ T. Paiva and R. R. dos Santos, Phys. Rev. B 65, 153101 (2002); 62, 7007 (2000); 58, 9607 (1998).
${ }^{13}$ T. Paiva and R. R. dos Santos Phys. Rev. Lett. 76, 1126 (1996).

${ }^{14}$ T. Paiva, M. El. Massalami, and R. R. dos Santos, J. Phys.: Condens. Matter 15, 7917 (2003).

${ }^{15}$ D. Góra, K. J. Rościszewski, and A. M. Oleś, J. Phys.: Condens. Matter 10, 4755 (1998).

${ }^{16}$ M. Noh, D. C. Johnson, and G. S. Elliott, Chem. Mater. 12, 2894 (2000).

${ }^{17}$ L. Bauernfeind, W. Widder, and H. F. Braun, Physica C 254, 151 (1995).

${ }^{18}$ S. G. Ovchinnikov, Phys. Usp. 46, 21 (2003).

${ }^{19}$ S. R. White, Phys. Rev. Lett. 69, 2863 (1992); 77, 3633 (1993).

${ }^{20}$ A. L. Malvezzi, Braz. J. Phys. 33, 55 (2003).

${ }^{21}$ N. A. Lima, M. F. Silva, L. N. Oliveira, and K. Capelle, Phys. Rev. Lett. 90, 146402 (2003).

${ }^{22}$ N. A. Lima, L. N. Oliveira, and K. Capelle, Europhys. Lett. 60, 601 (2002).

${ }^{23}$ K. Capelle, N. A. Lima, M. F. Silva, and L. N. Oliveira, in The Fundamentals of Density Matrix and Density Functional Theory in Atoms, Molecules, and Solids, edited by N. Gidopoulos and S. Wilson (Kluwer, Dordrecht, 2003).

${ }^{24}$ The computational efficiency of BA-LDA is crucial for the production of Fig. 4, which requires for each value of $U_{i}$ many calculations with different values of $v_{i}$, until the $v_{i}$ that smoothes the density or equalizes the energy is found.

${ }^{25}$ Improved LDA functionals, currently under construction, have in preliminary calculations shown even smaller deviations from DMRG data.

${ }^{26}$ For visual clarity, Figs. 2-4 contain only curves obtained with BA-LDA, but as Fig. 1 and Table I show, the difference between the DMRG and DFT/BA-LDA results is much smaller than the difference between the effect of $U_{i}$ and that of $v_{i}$, which is robust and not dependent on computational methodology. 
${ }^{27}$ Note that one cannot use the Hohenberg-Kohn theorem to argue that the potential that produces the same density distribution in both systems must be the same one that yields the same groundstate energy. The ground-state energy is a unique functional of the density only for fixed interaction, whereas in the present case the two superlattices that are being compared have different interactions. Hence the two criteria are different, and the two sets of potentials in Fig. 4 are not identical.

${ }^{28}$ K.C. thanks Ferdinand Evers and Erik Koch for useful discussions of this issue. 